\title{
Long Range Obstacle Detection Using Laser Scanner and Stereovision
}

\author{
Mathias Perrollaz, Raphaël Labayrade, Cyril Royère, Nicolas Hautière, Didier Aubert \\ LIVIC (INRETS / LCPC) \\ Bât. 824, 14, route de la Minière \\ 78000 Versailles - France \\ mathias.perrollaz@inrets.fr
}

\begin{abstract}
To be exploited for driving assistance purpose, a road obstacle detection system must have a good detection rate and an extremely low false detection rate. Moreover, the field of possible applications depends on the detection range of the system. With these ideas in mind, we propose in this paper a long range generic road obstacle detection system based on fusion between stereovision and laser scanner. The obstacles are detected and tracked by the laser sensor. Afterwards, stereovision is used to confirm the detections. An overview of the whole method is given. Then the confirmation process is detailed: three algorithms are proposed and compared on real road situations.
\end{abstract}

\section{INTRODUCTION}

Perceptive systems are now a very useful function for the development of ADAS (Advanced Driving Assistance Systems). Particularly, road obstacle detection is necessary for various tasks such as pre-crash, collision mitigation, stop\&go, obstacle avoidance or inter-distance management. For this purpose, radar is a frequently cited sensor [12]. Laser scanner is also widely studied [3]. Even if both sensors are quite accurate, they provide only an incomplete representation of the scene. Thus the scope of such a solution is limited.

In parallel, a lot of studies concern vision systems. Monovision has been widely investigated [1] [2]. Currently, stereovision is more and more used, because it can produce a complete three dimensional view of the scene. In this field, various approaches have been proposed, focusing for example on the road geometry [5] or on the detection of obstacle points [9]. Stereovision technics are computationally costly and thus a compromise between detection range and accuracy must be chosen.

To ensure a maximum reliability to the obstacle detection systems, many researches focus on multi-sensor fusion approaches [8] [11]. In particular, using stereovision and a laser scanner seems to be an efficient solution [6]. In order to provide better performances with such a configuration, we propose in this paper an innovative fusion scheme. In this approach, the obstacle detection and tracking tasks are performed thanks to the laser scanner. The stereovision is used subsequently to confirm the detections. Perception range enhancement technics are included in the confirmation task. We will show in this paper that this strategy is highly reactive and reliable.

In section II, the configuration of the sensors is described. An overview of the obstacle detection system is then presented in section III. Section IV deals with the stereovision stage of the algorithm. Then, three obstacle confirmation strategies are compared in real road conditions in section $\mathrm{V}$.

\section{SEnsor CONFIGURATION}

The geometrical configuration of the used sensors is described on Fig. 1. To easily merge data from both sensors,

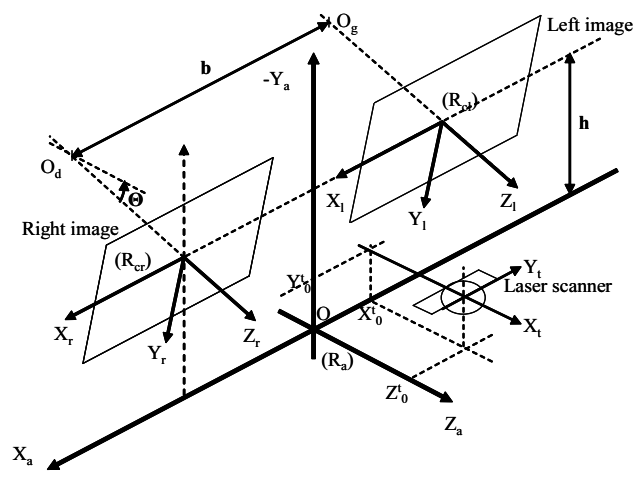

Fig. 1. Geometry of the stereoscopic and laser sensors.

let us define an absolute coordinate system $R_{a}$ linked to the vehicle. The laser scanner is rigidly linked to $R_{a}$, and placed with its detection plane parallel to the $\left(O, X_{a}, Z_{a}\right)$ plane. So, the coordinates of a laser point $\left(X_{t}, Y_{t}\right)$ can be easily calculated in $R_{a}$ :

$$
\left\{\begin{aligned}
X_{a} & =-Y_{t}-X_{0}^{t} \\
Y_{a} & =Y_{0}^{t} \\
Z_{a} & =X_{t}+Z_{0}^{t}
\end{aligned}\right.
$$

where $\left(X_{0}^{t}, Y_{0}^{t}, Z_{0}^{t}\right)$ are the coordinates of the laser scanner in $R_{a}$.

In addition, we use a stereoscopic sensor placed behind the vehicle windshield. The image planes are almost aligned. The epipolar geometry is rectified after calibration, so that the epipolar lines are parallels. Cameras are described by a pinhole model and characterized by $\alpha=\frac{\text { focal length }}{\text { pixel size }}$ (pixels are considered as squared). The other parameters of the stereoscopic sensor are:

- $h$ : height of the cameras, 
- $\theta$ : pitch of the cameras,

- $b$ : the stereo baseline.

Given a point $P\left(X_{a}, Y_{a}, Z_{a}\right)$ in the absolute coordinate system $R_{a}$, its position in the stereoscopic images systems $\left(u_{r}, \Delta, v\right)$ and $\left(u_{l}, \Delta, v\right)$ can be calculated as:

$$
\left\{\begin{array}{lll}
u_{r} & =u_{0}+\frac{\alpha X_{a}-\alpha b / 2}{\left(Y_{a}+h\right) \sin \theta+Z_{a} \cos \theta} \\
v_{r}=v_{l} & =\frac{\left[v_{0} \sin \theta+\alpha \cos \theta\right]\left(Y_{a}+h\right)+\left[v_{0} \cos \theta-\alpha \sin \theta\right] Z_{a}}{\left(Y_{a}+h\right) \sin \theta+Z_{a} \cos \theta} \\
\Delta & =\frac{\alpha b}{\left(Y_{a}+h\right) \sin \theta+Z_{a} \cos \theta}
\end{array}\right.
$$

with $\Delta=u_{l}-u_{r}$ representing the disparity of the considered pixel. Thus, using equations (1) and (2), a laser point can be easily projected in the stereoscopic images.

\section{OVERVIEW OF THE APPLICATION}

Our fusion scheme is presented on Fig. 2. It relies on the detection and tracking of the obstacles by the laser scanner and a subsequent confirmation by stereovision.

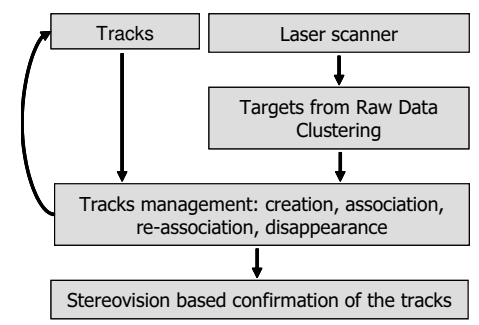

Fig. 2. Overview of the obstacle detection algorithm.

\section{A. Targets from the Laser Scanner}

The potential obstacles are detected using the laser scanner. This sensor scans an horizontal plane of the scene and provides a set of points (distances measured with centimetric precision). The main issue is the conversion of this raw data into a more symbolic representation. For this purpose, the data are first filtered to remove the laser points situated outside a warning area. This area can be build thanks to a road marker detector or to an inertial sensor used to predict the path of the vehicle. The set of potential obstacles is then created by clusterization of the remaining laser points. This step relies on a recursive nonsupervised automatic classification algorithm, which provides a set of obstacles. The obstacles are represented by ellipses, quantifying the uncertainty on their size and position. The processing of the laser data is described more extensively in [6].

\section{B. Tracks Management}

Obstacle detection using a laser scanner is sensitive to the vehicle pitch. Indeed pitch can make the laser plane cut the road surface or go over a far obstacle. Both cases lead to non detections. To solve this issue and avoid losing the obstacles, a multi-target association and tracking algorithm is applied to the ellipses representing the obstacles. Such a tracking is also necessary to determine the evolution of the perceived objects relative to our vehicle (relative speed, Time To Collision, ....). It is applied on laser data to beneficiate from high reactivity and accuracy. The used algorithm, based on Kalman filtering (tracking step) and belief theory (association step), is fully detailed in [6].

\section{Stereovision Based Confirmation of the Laser Tracks}

A major issue with an obstacle detection system using a single laser scanner is the abundance of false detections. Indeed, in case of strong pitch of the car or of non-plane road geometry, the road surface can be detected as an obstacle. This is illustrated on Fig. 3. Moreover, the errors in the tracking model can also lead to false detections. To solve these problems and ensure a maximum reliability to our system, we propose to use local stereovision to confirm (or infirm) the laser tracks a posteriori. The stereoscopic sensor can actually benefit from higher quantity of information than the laser scanner. So it can more easily distinguish the obstacles from the road surface. Now, let us explain more precisely the stereovision stage of the algorithm.
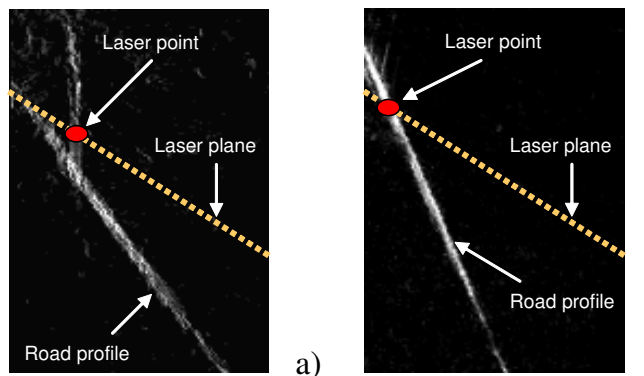

b)

Fig. 3. V-disparity view of the laser scanner plane. a) An obstacle is detected. b) The road is viewed as an obstacle, due to vehicle pitch.

\section{STEREOVISION BASEd CONFIRMATION OF AN OBSTACLE}

The stereovision based confirmation consists in four major steps:

- determination of regions of interest in the stereoscopic images,

- application of a numerical zoom to maximize the detection range,

- computation of a local disparity map in the regions of interest,

- criterion evaluation from this disparity map to confirm the existence of an obstacle. Three different approaches will be proposed and compared.

\section{A. Determination of the Regions of Interest}

The first step of the confirmation process consists in building regions of interest in the stereoscopic images. For this purpose, a bounding box $V_{o}$ is constructed in $R_{a}$ from the laser tracks as described on Fig. 4-a. $Z_{\text {near }}, X_{\text {left }}$ and 
$X_{\text {right }}$ are computed from the ellipse parameters. $Z_{f a r}$ and $Y_{\text {high }}$ are then constructed from an arbitrary knowledge of the size of the obstacles.
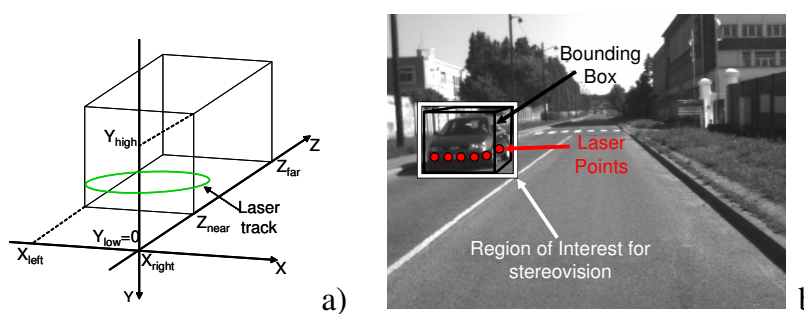

a)

b)

Fig. 4. Definition of a volume of interest for a laser track (a), and corresponding region of interest in the right image (b).

Given the $V_{0}$ volume, the regions of interest in both stereoscopic images (Fig. 4-b) are defined by calculating the coordinates of the summits of $V_{o}$ in the $\left(u_{r}, \Delta, v\right)$ and $\left(u_{l}, \Delta, v\right)$ spaces, using equation (2).

\section{B. Stereovision Range Enhancement Using a Numerical Zoom}

To ensure a real time system performance, our stereoscopic sensor uses quarter PAL images. Unfortunately, this resolution reduces the range of obstacles detection. The laser scanner can perform detection up to 75 meters, but the stereovision limits the system : it can hardly confirm further than 40 meters. Indeed, several disturbing elements appear at long ranges:

- The objects become small against the correlation windows. The risk of having objects of different disparities in the same correlation window is consequently increased. This can lead to errors in the disparity map.

- The number of pixels in the regions of interest becomes small. This gives more importance to the errors in the disparity map.

- Eventually, the dynamic in disparity is poor at long distances, making it more difficult to distinguish the obstacles from the road surface.

The use of images at higher resolution could increase the range so that the limiting sensor would not be the stereovision anymore. But the computation time would subsequently exceed our fixed limit of $40 \mathrm{~ms}$.

1) Principle: Our idea to solve the dilemma between range and computation time is to use a different resolution for each region of interest, depending on the distance measured by laser scanner. The different resolutions are obtained using numerical zoom with bicubic interpolation. As it is presented on Fig. 5, the magnification factor can be calculated to obtain a constant object size in the images, independently from the distance. Consequently, each object is observed and processed in a similar way. Only its level of blurredness limits the magnification factor.

The numerical zoom solution has double advantage: detecting far objects (zoom in) and saving computation time when

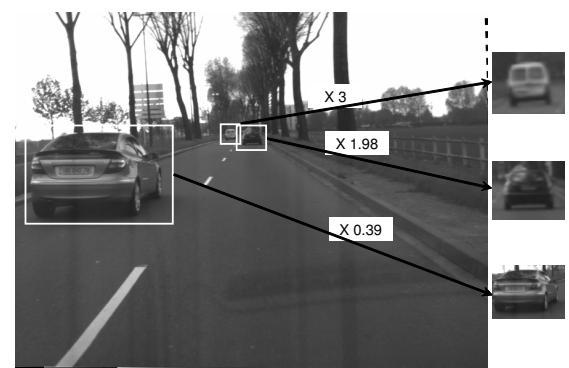

Fig. 5. Different zoom ratio applied to different obstacles to have sizes of objects which are independent from their distances.

dealing with near objects (zoom out).

2) Definition of the Magnification Factor: To compute the level of magnification that needs to be apply to a given region of interest, let us consider an object of width $L$ meters (see Fig. 6). We assume that this object is fronto-parallel to the image planes. $P 1$ and $P 2$ are projected respectively to $p_{1}\left(u_{r 1}, v, \Delta\right)$ and $p_{2}\left(u_{r 2}, v, \Delta\right)$.
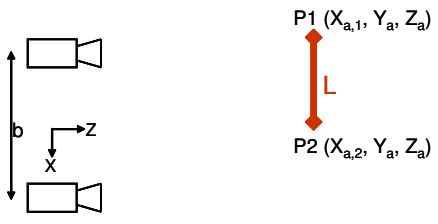

Fig. 6. Observed object, parallel to the images planes.

The object size in the zoomed image is attempted to be $n$ pixels:

$$
\begin{aligned}
n & =u_{r 1}^{z o o m}-u_{r 2}^{z o o m} \\
& =F_{z}\left(u_{r 1}-u_{r 2}\right)
\end{aligned}
$$

with $F_{z}$ the zoom ratio. Using equations (1) and (2), we can express $n$ as following:

$$
n=F_{z} \frac{\alpha L}{\left(Y_{0}^{t}+h\right) \sin \theta+\left(X_{t}+Z_{0}^{t}\right) \cos \theta}
$$

Furthermore, let us define a scale factor $K_{s}$ representing the ratio between the real size of an object and its desired size in the image:

$$
K_{s}=\frac{\text { Desired size in the image (pixels) }}{\text { Real size (m) }}
$$

$n$ must respect: $n=K_{s} * L$. So $F_{z}$ is finally given by:

$$
F_{z}=K_{s} \frac{\left(Y_{0}^{t}+h\right) \sin \theta+\left(X_{t}+Z_{0}^{t}\right) \cos \theta}{\alpha}
$$

\section{Construction of the Local Disparity Map}

All the proposed algorithms for obstacle confirmation (see IV-D) need to compute a local disparity map. Two different approaches have been tested for this purpose. One is funded on a correlation based stereovision algorithm, whereas the other uses a more original disparity propagation algorithm. 
1) Correlation Based Stereovision: The first algorithm is a classic correlation using ZNSSD (Zero Mean Sum of Squared Differences) criterion with a winner take all (w.t.a) strategy [10]. This score has the advantage to be centered on the average of pixel intensities and normalized by the standard deviation of the pixel intensities in the correlation windows. This makes it more robust against the additive and multiplicative differences of illumination between both cameras.

Only the pairs that present a strong similarity are considered as correspondent. In addition, the general error filtering [4] and the crossed-validation are also implemented to limit the errors and the influence of the semi-occluded areas. The perspective distortion of the road is also taken into account with a plane world homography similar to Wiliamson's approach [13]. A local disparity map computed with this algorithm is presented on Fig. 7-a.
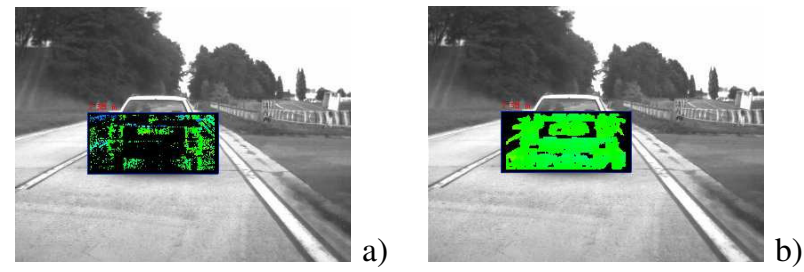

Fig. 7. Local disparity map computed using correlation algorithm (a) and disparity propagation (b).

2) Propagation of the Laser Points Disparity: Another matching solution is envisaged. It relies on the disparity propagation algorithm presented in [7]. It is well suited to obtain dense disparity maps in poorly textured regions such as road surface and vehicles. The originality of our approach consists in using the laser points as seeds and propagating their disparity in their neighborhood. Laser points are transformed into seeds $\left(u_{r}, \Delta, v\right.$, score $)$ using equations (1) and (2) and by calculating a correlation score. Fig. 7-b gives an example of the disparity map obtained.

The required knowledge of the laser points restricts the context of use of this method. Indeed, the laser points are present at the detection step but are unknown after the tracking algorithm. As a consequence, the use of this propagation algorithm must be limited to the laser targets or to the new tracks.

\section{Obstacle Confirmation Criteria}

To confirm the existence of an obstacle in a region of interest, three approaches are proposed.

1) Number of Obstacle-Pixels: The first approach consists in classifying the pixels of the region of interest (Fig. 8). A local road profile is first extracted using the $v$-disparity projection [5]. Afterwards, the $\left(u_{r}, \Delta, v\right)$ coordinates of each pixel are analyzed to determine whether it belongs to the road surface. If not, the pixel is classified as an obstacle-pixel. At the end of this process, every pixel in the region of interest has been classified as road or obstacle. The number of obstacle-pixels gives a confidence on the existence of an object over the road surface. Therefore, an obstacle is confirmed if the confidence is above a threshold.

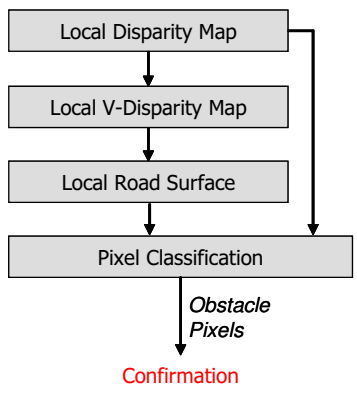

Fig. 8. Obstacle-Pixels algorithm for obstacle confirmation.

The obstacle-pixels criterion has the advantage to avoid any assumption on the obstacles to detect. Moreover, this method gives a confidence, in an intuitive way. However, as it considers each pixel individually, it can be strongly influenced by errors in the disparity map.

2) Prevailing Alignment Orientation: Assuming that the obstacles are seen as vertical planes by the stereoscopic sensor, an other confirmation criterion can be defined (Fig. 9). The prevailing alignment of pixels in the local $v$-disparity image is extracted using the Hough transform. The confirmation of the track depends on the orientation of this alignment: a quite vertical alignment corresponds to an obstacle. Other alignments correspond to the road surface.

The Prevailing Alignment criterion relies on a global

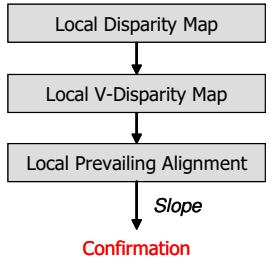

Fig. 9. Prevailing alignment algorithm for obstacle confirmation.

approach in the region of interest (alignment seeking). This makes it more robust with respect to the errors in the disparity map.

3) Laser Points Altitude: As explained in section III, many false detections are due to the intersection of the laser plane with the road. The knowledge of the longitudinal road geometry allows to deal with such errors. Therefore, the local profile of the road is estimated through $v$-disparity approach. The altitude of the laser points is then compared to the altitude of the local road surface. An obstacle is confirmed if 
this altitude is high enough (Fig. 10).

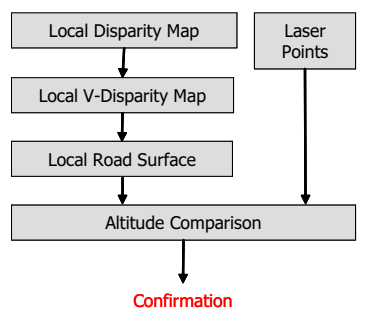

Fig. 10. Laser Points Altitude algorithm for obstacle confirmation.

This solution has the same advantages as the Prevailing Alignment criterion, but its context of use is restricted to the same situations as the disparity propagation algorithm (see IV-C.2).

\section{EXPERIMENTAL VALIDATION}

We have implemented the algorithms on one of the experimental vehicle of LIVIC to assess their behavior in real road conditions. The stereoscopic sensor is composed of two Sony ${ }^{T M} 8500 \mathrm{C}$ cameras with Computar ${ }^{T M}$ Auto Iris $8.5 \mathrm{~mm}$ focal length. Quarter PAL 8 bits gray-scale images are grabbed every $40 \mathrm{~ms}$. The baseline is $b=1 \mathrm{~m}$, the height $h=1.4 \mathrm{~m}$ and the pitch $\Theta=5^{\circ}$. The laser sensor is a $\operatorname{Sick}^{T M}$ scanner which measures 201 points every $26 \mathrm{~ms}$, with a scanning angular field of view of $100^{\circ}$. It is positioned horizontally $40 \mathrm{~cm}$ over the road surface. The whole algorithm runs at video frame rate on a dual IntelXeon ${ }^{T M} 1.8 \mathrm{GHz}$ personal computer.

Our main objective is to obtain a correct detection rate and almost no false detections.

Several aspects must be highlighted: the global performances (rates of non detections and false detections), the robustness of the criteria with respect to errors in the local disparity map, the detection range and the ability to work with various types of obstacles.

\section{A. Comparison of the Confirmation Criteria}

1) Detection Range and Influence of the Numerical Zoom: To quantify the detection range of our perception system and check the advantages of the numerical zoom, our experimental vehicle is placed in a fixed position on a 80 meters straight lane. Another vehicle slowly moves away. The distance where the perception system loses the target is measured. The results of this test are reported in Table I.

TABLE I

Detection Range of the Confirmation Algorithms and INFLUENCE OF THE NUMERICAL ZOOM.

\begin{tabular}{|l|c|c|c|c|}
\cline { 2 - 5 } \multicolumn{1}{c|}{} & $\begin{array}{c}\text { Laser } \\
\text { scanner }\end{array}$ & $\begin{array}{c}\text { Obstacle } \\
\text { Pixels }\end{array}$ & $\begin{array}{c}\text { Prevailing } \\
\text { Alignment }\end{array}$ & $\begin{array}{c}\text { Laser Points } \\
\text { Altitude }\end{array}$ \\
\hline Zoom off & $74 \mathrm{~m}$ & $31 \mathrm{~m}$ & $58 \mathrm{~m}$ & $74 \mathrm{~m}$ \\
\hline Zoom on & $74 \mathrm{~m}$ & $56 \mathrm{~m}$ & $74 \mathrm{~m}$ & $74 \mathrm{~m}$ \\
\hline
\end{tabular}

Without applying the zoom, the stereovision stage clearly limits the detection range of the system. Indeed, although the laser scanner detects the car up to 74 meters, the target is confirmed at 58 meters in the best case using Prevailing Alignment criterion. Obstacle-pixels criterion gives poor results beyond 31 meters. Only the laser points altitude criterion seems to give good results. But this is quite artificial. Indeed, no local road profile can found at long range because of the very little number of pixels in the region of interest. So the default profile (which is the actual one since our vehicle is in its resting position) is taken into account, thus the obstacle is confirmed.

The numerical zoom allows to increase the range. With prevailing alignment and laser points altitude criteria the detection range of our system is no longer limited by the stereoscopic sensor. Only the obstacle-pixels criterion can not confirm obstacles up to 74 meters, but the range is increased by $80.6 \%$.

Numerical zoom remains activated for the following tests.

2) False Detections: To assess the false detection rate, we drove on a very bumpy and dent parking area to obtain a large number of false detections due to the intersection of the laser plane with the road surface. The results are reported in Table II (7032 images have been processed).

TABLE II

FALSE DETECTIONS.

\begin{tabular}{|c|c|c|c|c|}
\cline { 2 - 5 } \multicolumn{1}{c|}{} & $\begin{array}{c}\text { Laser } \\
\text { scanner }\end{array}$ & $\begin{array}{c}\text { Obstacle } \\
\text { Pixels }\end{array}$ & $\begin{array}{c}\text { Prevailing } \\
\text { Alignment }\end{array}$ & $\begin{array}{c}\text { Laser Points } \\
\text { Altitude }\end{array}$ \\
\hline false detections & 781 & 3 & 10 & 167 \\
\hline
\end{tabular}

False detections are globally correctly invalidated using the obstacle-pixels and prevailing alignment criteria. The laser points altitude criterion provides more false detections than expected, because of its high sensibility to the calibration errors between stereovision and laser scanner. Indeed, a slight error in the positioning of the scanner relative to the cameras can lead to a serious error in laser points projection, especially at long ranges. The other criteria are not dramatically affected by this issue.

Most of the remaining false detection occur when the local road surface is uniform, without any texture allowing to match pixels. So they can be removed using simple heuristics as: no obstacle can be confirmed without enough information in the region of interest. It hardly affects the detection rate, and the false detection rate of obstacle-pixels criterion almost falls to zero.

The main source of errors for the prevailing alignment algorithm comes from cases where the road surface has non relevant texture, but where the bounding box contains a small part of a nearby object (wall, vehicle,...).

3) Detection Failure: We have evaluated the rate of correct laser detections that have been confirmed by the different criteria. To check, at the same time, that it can indifferently deal with various kinds of obstacles, this test has been 
realized with two different obstacles: a vehicle followed by the instrumented vehicle (1268 images processed), and a pedestrian crossing the road at various distances (1780 images processed). The confirmation rate of each criterion (number of obstacles detected by the laser / number of obstacles confirmed) for these two scenarios is reported in Table III.

The three criteria can successfully confirm most of the

TABLE III

RATE OF CORRECT DETECTIONS SUCCESSFULLY CONFIRMED.

\begin{tabular}{|c|c|c|c|}
\cline { 2 - 4 } \multicolumn{1}{c|}{} & $\begin{array}{c}\text { Obstacle } \\
\text { Pixels }\end{array}$ & $\begin{array}{c}\text { Prevailing } \\
\text { Alignment }\end{array}$ & $\begin{array}{c}\text { Laser Points } \\
\text { Altitude }\end{array}$ \\
\hline Car & $97.4 \%$ & $98.5 \%$ & $95.2 \%$ \\
\hline Pedestrian & $91.9 \%$ & $94.9 \%$ & $97.8 \%$ \\
\hline
\end{tabular}

detections with both kinds of obstacles.

4) Conclusion of the Comparison: None of the presented obstacle confirmation criteria really outperforms the others. The obstacle-pixels is based on an intuitive approach and can deal with any types of obstacles. But it is seriously influenced by the quality of the disparity map. The more global feature of the prevailing alignment criterion makes it more robust to this kind of errors.

The laser points altitude is not sufficiently reliable to be exploited alone. Thus an efficient architecture for the application consists in using the laser points altitude to invalidate some false laser targets before the tracking step. Then the tracked obstacles are confirmed using obstacle-pixels criterion.

\section{B. Influence of the Matching Criteria}

Most of the errors in the confirmation process occur when the amount of information in the regions of interest is too low. To build a more dense disparity map in this case, we proposed to use the disparity propagation algorithm. Using this algorithm for the same tests as earlier highlights three aspects: Firstly, this method can enhance the performances of the confirmation stage on some poorly textured surfaces. But the propagation sometimes generates some correlated errors, which are very disturbing for our criteria. At last, this method is very sensitive to the calibration errors between stereovision and laser scanner.

\section{CONCLUSION AND OUTLOOK}

We have presented in this paper an innovative method for long range road obstacles detection (exemples of detections are given on Fig.11). In particular, we presented and compared three obstacle confirmation criteria which have distinct advantages and drawbacks. In parallel, a range enhancement technic using numerical zoom has been presented. It is efficient and allow to take advantage of higher resolution images without increasing the computation time.

The whole system is successfully used in the LIVIC experimental vehicles for collision-mitigation purpose. This system has very good performances and the remaining false alarms (two for $500 \mathrm{~km}$ driving) are due to very complex urban situations. Thanks to the range enhancement, it is also possible to
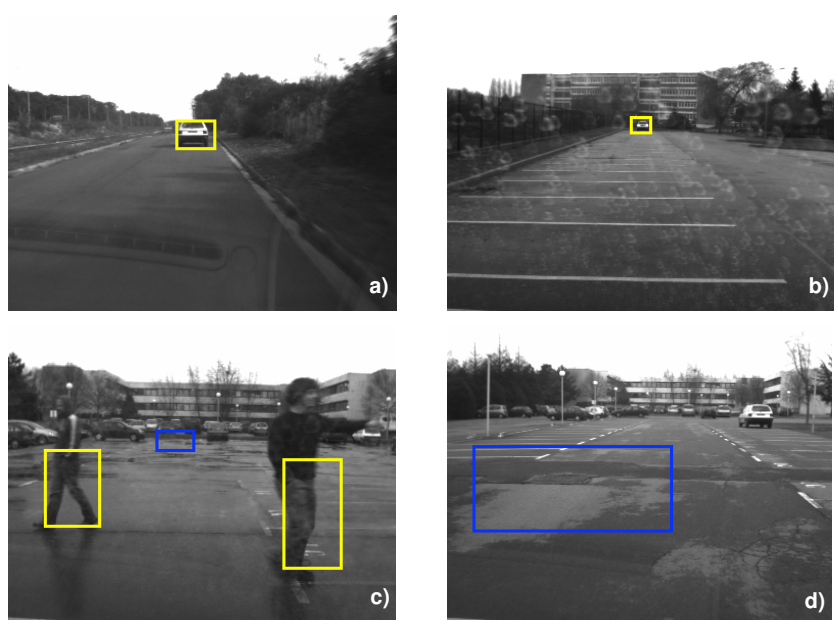

Fig. 11. Exemples of detections (yellow targets are confirmed, blue ones are rejected): a) a car $25 \mathrm{~m}$ away, b) a car $72 \mathrm{~m}$ away, c) two pedestrians, d) a false detection is rejected.

develop stop \& go and interdistances management applications for urban highway. The performances of this last systems are currently assessed.

Future work will deal with the possible combination of various confirmation and matching criteria.

\section{REFERENCES}

[1] M. Betke, E. Haritaoglu, and L. S. Davis. Multiple vehicle detection and tracking in hard real time. Technical Report CS-TR-3667, 1996.

[2] V. Graefe and W. Efenberger. A novel approach for the detection of vehicles on freeways by real-time vision, 1996.

[3] J.A. Hancock. Laser Intensity-Based Obstacle Detection and Tracking. $\mathrm{PhD}$ thesis, Carnegie Mellon University, 1999.

[4] H. Hirschmüller, P.R. Innocent, and J.M. Garibaldi. Real-time correlation-based stereo vision with reduced border errors. International Journal of Computer Vision, 47(1-3):229-246, 2002.

[5] R. Labayrade, D. Aubert, and J.P. Tarel. Real time obstacle detection on non flat road geometry through ' $\mathrm{v}$-disparity' representation. In Proceedings of IEEE Intelligent Vehicle Symposium, Versailles, France, 2002.

[6] R. Labayrade, C. Royere, D. Gruyer, and D. Aubert. Cooperative fusion for multi-obstacles detection with the use of stereovision and laser scanner. Accepted in the special issue of "Autonomous Robots", on "Robotics Technologies for Intelligent Vehicles", 2005.

[7] M. Lhuillier and L. Quan. Robust dense matching using local and global geometric constraints. In Proceedings of the 16th International Conference on Pattern Recognition, Barcelona, Spain, volume 1, pages 968-972, 2000.

[8] R. Mobus and U. Kolbe. Multi-target multi-object tracking, sensor fusion of radar and infrared. In Proceedings of IEEE Intelligent Vehicle Symposium, Parma, Italy, 2004.

[9] S. Nedevschi, R. Schmidt, and al. High accuracy stereo vision system for far distance obstacle detection. In Proceedings of IEEE Intelligent Vehicle Symposium, Parma, Italy, 2004.

[10] D. Scharstein and R. Szeliski. A taxonomy and evaluation of dense two-frame stereo correspondence algorithms. International Journal of Computer Vision, 47(1-3):7-42, 2002.

[11] B. Steux. Fade: A vehicle detection and tracking system featuring monocular color vision and radar data fusion. In Proceedings of IEEE Intelligent Vehicle Symposium, Versailles, France, 2002.

[12] S. Sugimoto, H. Tateda, H. Takahashi, and M. Okutomi. Obstacle detection using millimeter-wave radar and its visualization on image sequence. In IAPR ICPR, Cambridge, UK, 2004.

[13] T. Williamson. A High-Performance Stereo Vision System for Obstacle Detection. PhD thesis, Carnegie Mellon University, 1998. 